\title{
GESTIÓN ESTRATÉGICA E INNOVACIÓN EN LAS PYMES PERUANAS BAJO EL ACTUAL CONTEXTO DE CRISIS ECONÓMICA INTERNACIONAL
}

\author{
Francisca Bouby Tolentino* \\ f_bouby@yahoo.com
}

\begin{abstract}
RESUMEN
El presente trabajo tiene por objetivo plasmar el reto que significa para las Pequeñas y Micro empresas (PYME) peruanas, en términos de gestión e innovación, hacer frente a la alta competencia en una economía abierta; teniendo como marco un contexto de la crisis financiera internacional que se inició en el año 2008 y que aún no termina, lo que reafirma la tendencia cíclica del capitalismo, el gran potencial desestabilizador del sistema financiero actual y la alta competencia que afecta la competitividad de cualquier economía, con grandes impactos para las economías desarrolladas y en desarrollo, específicamente en la economía peruana donde ya empiezan a detectarse sus primeros efectos.
\end{abstract}

Palabras claves: Crisis, Gestión, Innovación, Creatividad, Tecnología.

\section{ABSTRACT}

This paper wants to highlight the meaning for small Peruvian enterprises , in terms of management and innovation, to face a high level of competition in an open economy; but having as a framework the current international financial crisis which began in 2008 but seems not to be over. This only reaffirms cyclical capitalism trend, and also the big power of current financial system and a high competition that affects the competitiveness of any economy. With great impacts on developed and non developed economies, mainly inside Peruvian economy where it began to detect its initial effects.

Keywords: Crisis, Management, Innovation, Creativity, Technology.

* Doctora en Economía por la UNAM, México, Magíster en Economía con mención en Política Económica por la UNMSM, Economista. Investigadora y docente a nivel de pre-grado y post-grado. 


\section{INTRODUCCIÓN}

El presente trabajo tiene por objetivo plasmar el reto que significa para las Pequeñas y Micro empresas (PYME) peruanas hacer gestión en una economía abierta de alta competencia teniendo como marco un contexto de crisis financiera internacional que se inició en el año 2008 y que aún no termina, con grandes impactos para las economías desarrolladas y en desarrollo, específicamente en América Latina y en la economía peruana donde ya empiezan a detectarse sus primeros impactos.

En el primer apartado se presenta el marco contextual donde se hace el análisis de la actual tendencia globalizadora que genera alta competencia por el reparto de los mercados entre las empresas transnacionales, cuya presencia en los países en desarrollo genera crecimiento económico, muy distante de lo que significa desarrollo económico de una nación.

En el segundo apartado se desarrolla el Marco Teórico, donde se presenta la discusión sobre la crisis financiera internacional que se inició en el 2008 y que plantea el desafío teórico y de interpretación del fenómeno de la crisis.

En el tercer apartado se presentan las características e importancia de las PYME en la economía peruana, destacándose el elevado nivel de informalidad existente en el sector empresarial privado peruano.

En el último apartado se destaca la necesidad de una gestión estratégica por parte del Estado para la innovación del sector empresarial en el Perú, principalmente de las PYME.

\section{MÉTODO}

El método empleado es el de análisis y síntesis. El diseño de la investigación empleado es el análisis de corte transversal Las fuentes de información son básicamente secundarias, tanto fuentes oficiales nacionales e internacionales, así como trabajos de investigadores y autores de reconocida trayectoria.

\section{DISCUSIÓN TEÓRICA}

\section{Marco contextual}

El contexto actual se caracteriza por un proceso de globalización e integración económica, y por la crisis financiera internacional que involucra tanto a los países desarrollados como a los países en desarrollo. a. Globalización económica: elementos de la nueva geopolítica mundial

El actual proceso de globalización tiene elementos que configuran una nueva geopolítica mundial por la hegemonía de los mercados. Son elocuentes las palabras Seitz, Director de Planificación Estratégica de Alemania, quien señala que “... la guerra económica global es por la repartición de la riqueza planetaria entre los poderosos. Antes se hacían guerras por territorios hoy se hace por mercados ..." (Contreras, 2002).

La globalización económica es un proceso liderado por las grandes empresas transnacionales, bajo un contexto de alta competencia, orientado fundamentalmente a la recomposición de la acumulación del capital. Al respecto, Chomsky señaló a mediados de los 90: "De las diez compañías más grandes del mundo, seis son transnacionales japonesas, tres son estadounidenses y una es británica/holandesa. La distribución geográfica de las corporaciones refleja las habituales estructuras de poder en la sociedad global: 435 de las 500 transnacionales más importantes pertenecen a los países del Grupo G-7. De ellas 151 son estadounidenses, 149 japonesas, 44 alemanas, 40 francesas, 33 británicas, 11 italianas y 5 canadienses”. (Contreras, 2002).

Bajo este contexto de globalización, la presencia de estas empresas transnacionales en los países en desarrollo es creciente y responde a la necesidad de reducir costos en la elaboración de los bienes o servicios. Al respecto, el empresario inglés Barnevik manifiesta; “... tenemos posiciones en Norte América, Europa Oriental y Asia. ... Ahora tenemos que explotarlos. El mensaje de crecimiento nos está dando impulso ..."(Contreras, 2002:122).

Precisando que el proceso de la transnacionalización si bien es cierto genera crecimiento económico, sin embargo, este concepto dista mucho de lo que significa desarrollo económico de una nación.

A las empresas transnacionales les interesa solamente el crecimiento; no así si un país logra o no avanzar en su desarrollo, por lo que la actitud y la conducta del empresario de las transnacionales expresado en las palabras de Beck, son: "En primer lugar, podemos exportar puestos de trabajo allí donde sean más bajos los costos laborales y las cargas fiscales a la creación de mano de obra" (Contreras, 2002: 18). 
El neoliberalismo, al promover el libre mercado, asegura también la libertad de movimiento de los factores productivos, a decir de Garnier: “...el mercado es el instrumento más poderoso para la toma ágil, barata, transparente, eficiente de decisiones privadas e individuales. En el mercado, los costos y los beneficios se traducen por medio de los precios: uno sabe cuánto cuesta, cuánto le gusta y por eso es un extraordinario mecanismo de coordinación de la vida social" (Contreras, 2002).

En el contexto de la economía de mercado los países en desarrollo, a fin de atraer la inversión, se proyectan bajo el concepto marca-país, etiquetándose lo que no son, tal como lo señala Yory: “ ... disfrazando sus miserias, desigualdades, discriminaciones e inequidades ..." (Yory, 2012)

En el caso de los países denominados BRICS (Brasil, Rusia, India, China y Sudáfrica), se le ha etiquetado como el grupo que “ ... asumirá el liderazgo de la economía mundial” hacia el 2050. Lo que no resulta claro es cuál va a ser el beneficio para la mayor parte de la población de los países que pertenecen a este grupo, sobre todo en los casos de India y Brasil donde las diferencias y desigualdad son más acentuadas. (Yory, 2012: 42).

\section{b. La crisis financiera global se inicia en el año 2008}

La crisis financiera global tiene efectos de concentración de la riqueza y de polarización de la sociedad.

La globalización financiera propició que el sector financiero se erija como eje de acumulación del sistema capitalista, al permitir que en operaciones internacionalmente interconectadas se origina sobreliquidez muy por encima del valor de los capitales invertidos generándose, como consecuencia de las fuerzas del mercado, inestabilidad y especulación, lo cual condujo a la crisis financiera internacional que corrobora la tendencia cíclica del sistema y el poder desestabilizante del sistema financiero actual, especialmente en Estados Unidos y la Unión Europea. (Huerta Moreno, 2013: 83).

Las consecuencias de la crisis son divergentes: enormes ganancias para quienes tomaron riesgos en el mercado de capitales y sistemas bancarios, apoyados por sus gobiernos paralelamente enormes costos socio-económicos para el resto de la población estadounidense y europea lo que viene afectando fuertemente el estado de bienestar de las clases medias y de los trabajadores empobrecidos por cuanto no reciben el apoyo de parte de sus gobiernos y que, a diferencia de los banqueros, son los sectores que están cargando con los costos de la recomposición de las relaciones financieras y la restauración de la liquidez, lo cual tiene efectos polarizantes en la sociedad. (Huerta Moreno, 2013: 83)

El Fondo Monetario Internacional considera que si Estados Unidos o Europa continúan recesión los precios de las materias primas caerán afectando a los países de la región latinoamericana y señala que en este contexto, países como Perú deben prepararse para un escenario negativo y aplicar, cuanto antes, políticas para disminuir el impacto.

\section{II: Marco teórico}

La crisis financiera del 2008 marca el inicio de la depresión que afecta principalmente a Estados Unidos y Europa que, si bien no tiene el nivel de profundidad de la crisis de 1929, sin embargo mantiene ciertas características como el enorme desempleo generado y con ello la disminución de la calidad de vida de la población, y la creciente desigualdad que se está generando al concentrarse la riqueza cada vez más en un porcentaje minoritario de la población.

La bibliografía existente sobre la crisis es basta y se orienta a explicar fundamentalmente el por qué ha surgido esta crisis y cuáles son las consecuencias. Un aporte valioso al respecto lo presenta Stiglitz, Premio Novel de Economía 2001.

Otro aporte valioso lo da Krugman, Premio Novel de Economía 2008, en su última publicación del 2012 "Detengamos esta crisis, Ya!" donde se orienta a dar respuesta a la pregunta y ahora qué hacemos. Ambos autores pertenecen a la escuela neokeynesiana.

\section{a. ¿Porqué surgió la crisis?}

Para dar respuesta esta pregunta Stiglitz (2012) parte del análisis de uno de los principios generales de la teoría económica: la mano invisible de Adam Smith, quien es considerado como el padre de la teoría económica moderna. Smith sostenía al buscar su propio interés que la iniciativa privada generaría, como a través de una mano invisible, el bienestar de todos. 
La explicación teórica de por qué surgió la crisis, según Stiglitz, es que los incentivos de los banqueros no estaban bien alineados con la rentabilidad social. Sostiene "Cuando los mercados funcionan bien -de la forma postulada por Adam Smith- es porque la rentabilidad privada y los beneficios sociales están bien alineados...", tal como sostenía la teoría de la productividad marginal, según la cual “... la contribución social de cada trabajador es exactamente igual a su remuneración privada. Las personas con una productividad más alta -con una contribución social mayor-reciben un salario más alto..." (Stiglitz, 2012: 68).

Un supuesto fundamental de esta teoría es la existencia de competencia perfecta entre las empresas. Sin embargo, el mundo actual globalizado ya no se sostiene en dicho supuesto; la situación ha cambiado radicalmente por la presencia de las empresas transnacionales que se mueven bajo un nuevo supuesto: el de la competencia imperfecta.

\section{b. ¿Cuáles son las consecuencias de la crisis?: desigualdad e injusticia}

Para dar respuesta a esta pregunta, Stiglitz cuestiona el papel de los mercados, al sostener que su fracaso se debe a su ineficiencia, cuestionando así una de las leyes más elementales de la teoría económica: que la demanda se iguale a la oferta.

Afirma Stiglitz que vivimos en un mundo en el cual hay grandes necesidades insatisfechas -muestra de ello es la miseria existente en los países más pobres de África y otros continentes; la incapacidad de afrontar los desafíos del calentamiento global, además de la infrautilización de los principales factores de la producción, lo que genera un enorme desempleo que, a decir de Stiglitz, es la peor falla del mercado “... la principal fuente de ineficiencia y una importante causa de la desigualdad-" (Stiglitz, 2012:21).

Sin embargo, Stiglitz lo justifica así: “... El problema no es que la globalización sea mala o injusta, sino que los gobiernos la están gestionando de una forma muy deficiente -mayoritariamente en beneficio de intereses especiales- ...". Lo mismo, argumenta a favor de la economía de mercado, “... el poder de los mercados es enorme, pero no poseen un carácter moral intrínseco. Tenemos que decidir cómo hay que gestionarlos... " (Stiglitz, 2012:22).

Para Stiglitz, "La crisis financiera desencadenó una nueva conciencia de que nuestro sistema eco- nómico no solo era ineficiente e inestable, sino también básicamente injusto" (Stiglitz, 2012:23). Además, argumenta “... La riqueza que recibían la élites y los banqueros parecía surgir de su capacidad y su voluntad de aprovecharse de los demás" (Stiglitz, 2012:24). Sin embargo, concluye que si el gobierno estadounidense no señala a los culpables de haber llevado a la economía al borde de la crisis, y los deja en total libertad "quiere decir que el problema está en el sistema económico y político ..." (Stiglitz, 2012: 25)

Para Stiglitz, los apologistas de la desigualdad, defensores de la teoría económica del goteo, argumentan que dar más dinero a los de arriba beneficia a todo el mundo, vía crecimiento económico. Sin embargo, dice “... Lo que Estados Unidos ha venido experimentado durante los últimos años es lo contrario de la teoría económica del goteo: las riquezas que se han acumulado en lo alto se han producido a expensas de los de más abajo" (Stiglitz, 2012: 44).

Este razonamiento de Stiglitz le condujo a plantear que tanto el desplome de los empleos como la caída de los salarios producidos durante el último cuarto de siglo han profundizado la desigualdad generando una polarización en la sociedad estadounidense. Así, señala que “... aunque una mayor parte del dinero va a aparar a los de arriba, hay más gente que va hacia abajo" (Stiglitz, 2012: 45).

\section{Y ahora, ¿qué hacer?}

El análisis de Krugman parte de la pregunta: ¿por qué aumenta el desempleo y disminuye la producción? Su respuesta es porque la demanda es insuficiente (de los consumidores, empresarios y gobierno o $\mathrm{D}=\mathrm{C}+\mathrm{I}+\mathrm{G}+\mathrm{XN}$ ). Al estallar las burbujas gemelas de Estados Unidos y Europa, se hundió el gasto en construcción de viviendas $\left(\mathrm{C}_{\mathrm{bi}}\right)$ y en bienes de consumo $\left(\mathrm{C}_{\mathrm{bc}}\right)$; le siguió la inversión empresarial (I) al no tener el incentivo de las ventas para ampliar su capacidad productiva; y también ha caído el gasto de muchos gobiernos $(G)$ locales, estatales y algunos nacionales al encontrarse privados de muchos ingresos (T). De allí que Krugman afirme "Un gasto moderado, a su vez, implica una tasa de empleo moderada, porque las empresas no producirán lo que no pueden vender, y no contratarán a empleados si no los necesitan para la producción. Padecemos una grave falta de demanda, a escala global" (Krugman, 2012:35). 
Los indicadores que muestra la crisis son el bajo nivel de crecimiento del PBI y la enorme tasa de desempleo en Estados Unidos y Europa. Grecia, Irlanda y España presentan un desempleo del 23\% y de casi el 50\% entre los jóvenes (Krugman, 2012:9).

"Hay actores en el escenario político (con influencia real), que creen imposible que la economía en su conjunto pueda padecer una demanda insuficiente ... Sostienen que la gente tiene que gastar sus ingresos en algo" (Krugman, 2012:35).

Obviamente Krugman no cree que las cosas sean así y aduce que hay un rasgo crucial para comprender lo que ha fallado en la economía mundial, un rasgo que escapa, una vez tras otra, a la capacidad de comprensión de políticos y asesores.

¿Y, cuál es ese rasgo? Es el hecho de que "tu gasto es mi ingreso y mi gasto es tu ingreso". Esto es obvio pero no para las personas influyentes en el poder político. Por ejemplo, John Boehner, el presidente de la Cámara de Representantes de los Estados Unidos, influyó en el recorte presupuestal que hizo Obama (Krugman, 2012:40).

En los últimos 50 años la tarea de acabar con las recesiones ha sido cosa fundamental de la Reserva Federal que (a grandes rasgos) se ocupa de controlar la cantidad de dinero que circula en la economía; cuando la economía cae, la Reserva pone a trabajar la maquinita. Hasta antes de 2008 , esto siempre ha funcionado. Lo hizo muy bien tras la grave recesión de 1981-1982; también funcionó, aunque con más lentitud y titubeos, después de las recesiones de 1990-1991 y 2001 (Krugman, 2012:41).

Sin embargo, en la última crisis ya no ha funcionado. En realidad lo que controla la Reserva Federal es la base monetaria, el total de moneda que tienen los bancos, ya sea en circulación o en reserva. Y aunque la Reserva Federal ha triplicado la base monetaria desde el 2008, la economía sigue deprimida. ¿Significa esto que hay error cuando se afirma que hay un problema de demanda? No. De hecho, el fracaso de la política económica a la hora de resolver esta crisis era predecible dice Krugman (1999), ya que se ha llegado a una situación conocida como trampa de liquidez. ${ }^{1}$

A mediados de la década pasada, la economía estadounidense respondía a dos motores: la construcción inmobiliaria y el gasto de los consumidores. Ambos estaban siendo impulsados por el elevado precio de la vivienda, lo que condujo a una burbuja basada en expectativas poco realistas, y cuando ésta estalló arrasó con la construcción y el gasto de los consumidores, comenzando el declive de ambos en forma estrepitosa.

La Reserva Federal respondió con un rápido incremento de la base monetaria, teniendo para ello que rebajar la tasa de interés, tanto que pronto se llegó a una situación de trampa de liquidez.

Según Krugman, la economía se halla mutilada por la escasez de la demanda: "el sector privado, a nivel colectivo, intenta gastar menos de lo que gana, y la consecuencia es que los ingresos han caído. Pero estamos en una trampa de liquidez: la Reserva Federal ya no puede convencer al sector privado de que gaste más solo con aumentar la cantidad de dinero en circulación” (Krugman, 2012:48).

Y ¿cuál es la solución?: incrementar la demanda. Pero esta solución tan obvia, según Krugman, no es de la comprensión de las personas influyentes en las decisiones del gobierno. O también porque chocaría con los intereses económicos de ciertos grupos económicos fuertes que sí están siendo favorecidos por la situación de crisis en la economía.

\section{RESULTADOS: Características e importancia de las pymes en la economía peruana}

\section{Características de las pymes}

La legislación peruana define a la pyme como: “... la unidad económica constituida por una persona natural o jurídica, bajo cualquier forma de organización o gestión empresarial contemplada en la legislación vigente, que tiene como objeto desarrollar actividades de extracción, transformación, producción, comercialización de bienes o prestación de servicios ..." (Arbulú, 2006:32), debiendo contar con las siguientes características:

1. La trampa de la liquidez sucede cuando el Banco Central de Reserva (BCRP) que puede manejar la economía mediante su capacidad de alterar las tasas de interés, empieza a bajar la tasa de interés tanto que puede tocar el "límite inferior" de cero, situación en la cual tener el dinero bajo el colchón resulta ser mejor opción que prestarlo a otras personas. Es decir, en una situación de "trampa de la liquidez" ni siquiera una tasa de interés cero es lo suficientemente baja ya que el Banco Central de Reserva ha saturado la economía con liquidez hasta el punto en que tener más efectivo ya no supone ningún costo, pero la demanda general sigue siendo demasiada escasa.

En la actual crisis del sistema estadounidense la Reserva Federal pronto tocó el límite inferior: empezó a rebajar las tasas de interés a finales del año 2007, llegando cero a finales del 2008. Es más o menos lo que también ha ocurrido en Europa. 
- Tamaño. La microempresa tiene un número total de trabajadores entre uno y diez, y niveles de ventas anuales no mayores a 150 UIT.

La pequeña empresa tiene un número total de trabajadores hasta un máximo de cincuenta (50). de ventas anuales entre 51 y 85 UIT.

El sector informal comprende el autoempleo, las pymes y el trabajo doméstico, y se define como: “ ... el conjunto de unidades productivas de pequeño tamaño, con acceso limitado al capital, uso de tecnologías simples, poca división de trabajo y de la propiedad de los medios de producción y, generalmente operando en los márgenes del sistema legal institucional vigente" (Arbulú, 2006:33)

De acuerdo a la distribución por tamaño de las empresas formales e informales en el Perú, el 99,5\% de empresas son pymes formales e informales y el $97,9 \%$ son microempresas formales e informales. El nivel de informalidad es muy alto concentrándose en el sector de las pymes (Arbulú, 2006:36)

- Informalidad. Así, alrededor del 72\% del total de empresas no se hallan registradas en la Superintendencia Nacional de Aduanas y de Administración Tributaria (SUNAT). Casi el 75\% de las micro empresas son informales; alrededor del $30 \%$ de la pequeña empresa es informal; mientras que solo el $5 \%$ de la mediana empresa es informal y en la gran empresa, no existe informalidad. (Díaz y Kuramoto, 2012:21)

- Gestión empresarial. Alrededor del 64\% de las micro empresas declaró que sus negocios no tienen personería jurídica, cerca del 30\% tiene algún tipo de personería jurídica (destacando el caso de persona natural con negocio) y el $7 \%$ no tiene registro como empresa sino como independiente con recibo por honorarios profesionales. Más del $50 \%$ de los micro empresarios no tienen ningún tipo de registro contable, alrededor del $33 \%$ realiza apuntes personales y cerca del $20 \%$ tiene algún tipo de registro. (Arbulú, 2006:36)

\section{Participación de las PYME en la economía peruana}

La participación de las Pymes en la actividad económica productiva a nivel nacional es muy significativa, aunque con profundas limitaciones.

El sector de las PYME tiene gran importancia dentro de la estructura industrial del país, tanto en términos de su aporte a la producción nacional (49\% del PBI), como en relación a su potencial de absorción de mano de obra (cerca de $88 \%$ del empleo privado). Sin embargo, dado el alto nivel de informalidad existente en este sector, (que esconde el bajo nivel de capacitación de la mano de obra) la calidad del empleo es muy baja, lo que conduce a bajos niveles de productividad y competitividad, bajos niveles salariales y un elevado nivel de subempleo. (Arbulú, 2006:38))

Existen otros factores limitantes de la productividad tales como, la baja asociatividad y articulación con la mediana y gran empresa, bajo acceso al uso de las Tecnologías de la información y las comunicaciones (TIC) escaso y costoso acceso al financiamiento, baja capacidad de gestión empresarial, baja identidad y cultura empresarial y bajo nivel de acceso a nuevos mercados. (Giudice, 2010).)

Con estas profundas limitaciones, en la actualidad las PYME representan un estrato muy importante en la estructura productiva de la economía tienen una participación ampliamente mayoritria en los sectores productivos más importantes de la economía nacional constituyéndose en algunas zonas del interior del país como la única forma de organización empresarial existente sobre la cual gira la actividad económica.

Las PYME participan en el proceso productivo del país realizando un conjunto de actividades económicas heterogéneas, siendo comercio y servicios las que concentran la mayor cantidad de PYME (49\% y 33\%, respectivamente), seguido de manufactura (11\% del total de PYME formales) y más rezagados agropecuario y construcción (3\% cada uno). (Arbulú, 2006:6)

Las PYME concentran el $88 \%$ de los empleos del sector privado, generando 7,2 millones de puestos de trabajo. De éstos, la micro empresa genera 6,3 millones ( $77 \%$ de Población económicamente activa (PEA) ocupada en el sector privado a nivel nacional) y la pequeña empresa, 900.000 (dando trabajo al $11 \%$ de la PEA del sector privado).(Arbulú, 2006:6)

La estructura empresarial de la economía peruana se caracteriza por estar concentrada en micro empresas y un número relativamente menor de empresas medianas y grandes. Así, en el año 2006 existía un total de 3,2 millones de empresas en el sector privado, de las cuales, 3.167 .000 eran micro empresas, 50.000 eran empresas pequeñas, 


\section{Cuadro 1: PERÚ: PRINCIPALES VARIABLES, SEGÚN TAMAÑO DE EMPRESA}

2006

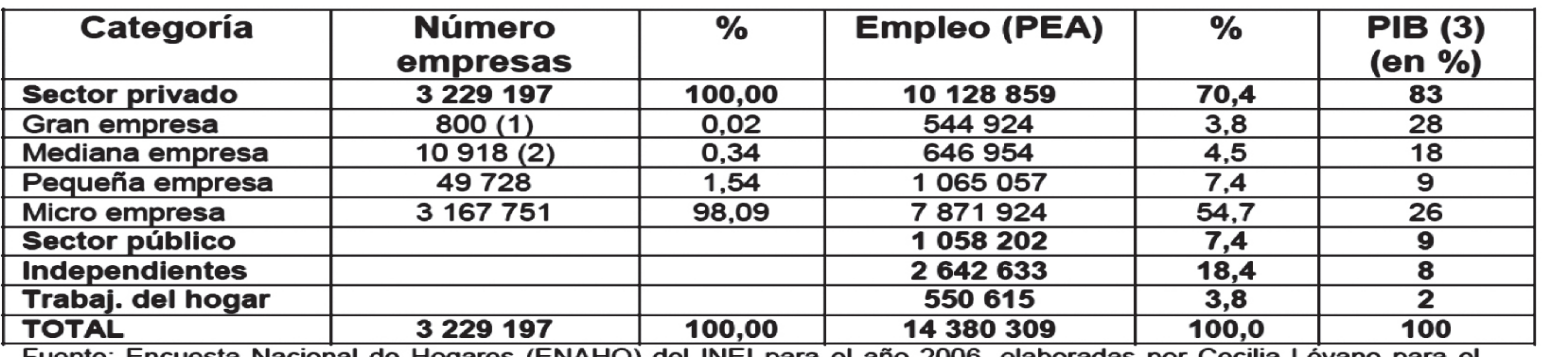

Fuente: Encuesta Nacional de Hogares (ENAHO) del INEI para el año 2006, elaboradas por Cecilia Lévano para el MTPE-Ministerio de Trabajo y Promoción del Empleo (2007)

10.000 empresas medianas, mientras que solo 800 eran empresas grandes. Vale decir que el $98 \%$ de las empresas del sector privado eran micro empresas, mientras que el $0,02 \%$ eran empresas grandes. (Villarán, 2012)

El total de micro empresas emplea al 55\% de la PEA ocupada y generan el 26\% del PBI, mientras que la gran empresa solo emplea a $3,8 \%$ de la fuerza laboral ocupada (ligeramente inferior al empleo generado por trabajadores del hogar) pero generan 30\% del PBI. Si agrupamos a las empresas grandes y las medianas, éstas emplean a $8,3 \%$ de la fuerza laboral ocupada del país y generan el $46 \%$ del PBI; mientras que el conjunto de las micro y pequeñas empresas emplean al $62 \%$ de la PEA ocupada y generan el 35\% del PBI. (Díaz y Kuramoto, 2012:21)

La innovación empresarial en el Perú: necesidad de una gestión estratégica para la innovación

Según Fernández Aceytuno, en materia de innovación, el grado es lo que marca la diferencia. ¿Y cuáles son los factores que pueden explicar esta diferencia? La respuesta es la estrategia. Dinamizar la innovación en las empresas requiere, primero que nada, dinamizar la forma en que éstas se gestionan.

Uno de las mayores limitantes del crecimiento económico en Perú son, entre otros, la calidad de la educación y la innovación. Según Inés Temple hay necesidad de invertir en educación del personal, a fin de repotenciarlos para mejorar su productividad y empleabilidad. Inés Temple, dice, “ ... una bomba de tiempo, ...nunca habrá paz social si no somos capaces de distribuir el conocimiento en forma equitativa". (BCRP, 2010).
- Innovación en las Pymes peruanas. La fuente de innovación en el Perú es aún externa debido a la compra de patentes. Por cada dólar invertido en innovación se necesita 10 US\$ en capacitación de personal. (Giudice, 2010:6).

La estructura empresarial heterogénea del sector privado en el Perú implica un reto al desarrollo de la innovación, la ciencia y la tecnología. En el caso de las micro empresas, existen grandes deficiencias en las habilidades y conocimientos, baja productividad y restricciones al financiamiento. Es ahí donde el Estado adquiere un rol importante en la formulación de políticas e instrumentos de política orientados a la reducción de la heterogeneidad del sector empresarial.(Díaz Y Kuramoto, 2010:22)

Son pocas las empresas que realizan actividades de ciencia y tecnología (ACT) en el Perú, siendo mayor las empresas de mayor volumen de ventas. Así, un 14\% de las mega empresas realizaron ACT en el 2004 , mientras que solo $4,9 \%$ de la grandes, $3 \%$ de las medianas, y $1,8 \%$ de las pequeñas realizaron este tipo de actividades. (Díaz y Kuramoto, 2010:52)

De otro lado, hay una clara desarticulación entre el sector industrial y las instituciones que generan conocimiento, lo cual se debe, por un lado, a la presencia de un sector empresarial con bajo nivel tecnológico como resultado de la poca presión competitiva a que estaba expuesto, lo cual no les obligaba a demandar servicios tecnológicos o a innovar permanentemente.

Actualmente, las empresas se hallan más expuestas a la presión competitiva del mercado externo, acentuado por la existencia de múltiples 
Tratados de Libre Comercio (TLC) a los cuales pertenece el Perú, lo que puede significar una ampliación de mercados, pero también una constante presión competitiva en base a calidad, precio e innovación; lo que implica, a su vez, la necesidad de contar con una logística eficiente que permita la entrega de los productos justo a tiempo y con sistemas de mercadeo adecuados a las exigencias del mercado externo. (Díaz y Kuramoto, 2010:66).

En cuanto a la legislación sobre política de ciencia, tecnología e innovación en Perú (CTI), ésta fue emitida partir de los 90, período en el que se inicia el proceso de estabilización de la economía y se reinicia el proceso de integración económica bajo la forma de los TLC.

Es a partir de este período que se inició la creación de varias instituciones orientadas a diseñar y ejecutar políticas de ciencia, tecnología e innovación, y se dictaron una serie de normas para dar sustento a dichas instituciones, como la Ley Marco de Ciencia, Tecnología e Innovación Tecnológica (Ley N. ${ }^{\circ}$ 28303), que define al Sistema Nacional de Ciencia, Tecnología e Innovación (SINACYT) y al Consejo Nacional de Ciencias, Tecnología e Innovación Tecnológica (CONCYTEC) como el ente rector del sistema.

Según Diaz y Kuramoto, la evolución de la política de ciencia, tecnología e innovación en el Perú ha pasado por tres etapas correspondientes a tres modelos de políticas de innovación: el modelo lineal o de primera generación; el modelo sistémico o de segunda generación; y el modelo de innovación generalizada o de tercera generación. (Díaz y Kuramoto 2010:86)². Pero es después de iniciado el período de estabilización económica que la política de CTI pasa de una visión de modelo lineal de innovación hacia una visión sistémica.

\section{CONCLUSIONES Y RECOMENDACIONES}

1. La actual tendencia globalizadora representa una guerra económica global por la repartición de los mercados.

2. La presencia de las empresas transnacionales en los países en desarrollo obedece a la necesidad de reducir costos en la elaboración de bienes o servicios.

3. La transnacionalización genera el crecimiento económico, algo muy distante de lo que significa desarrollo económico de una nación.

4. La trayectoria del crecimiento de la economía mundial sufre grandes oscilaciones causadas por recurrentes crisis en el sistema, como la generada en 2008 al convertirse el sector financiero en el eje de acumulación.

5. Con la crisis iniciada en el 2008 se reafirma la tendencia cíclica del capitalismo, el potencial desestabilizador del sistema financiero actual, y la alta competencia que afecta la competitividad de cualquier economía.

6. El sector empresarial peruano está conformado principalmente por micro y pequeñas empresas con capacidades tecnológicas sumamente limitadas. Así, alrededor del $72 \%$ del total de empresas no se hallan registradas en SUNAT. Casi el 75\% de las micro empresas son informales; alrededor del 30\% de la pequeña empresa es informal.

7. El alto nivel de informalidad existente en este sector esconde el bajo nivel de capacitación de la mano de obra, la calidad del empleo es muy baja, lo que conduce a bajos niveles de productividad y competitividad, bajos niveles salariales y un elevado nivel de subempleo

2 Díaz y kuramoto plantean tres políticos:

Las políticas de "primera generación" están basadas en la idea de un proceso lineal en la generación de innovaciones. Bajo esta idea, el proceso de innovación se inicia en los laboratorios científicos y mediante sucesivas etapas el conocimiento es incorporado en aplicaciones comerciales exitosas, y difundido en la economía.

Las políticas de "segunda generación" están basadas en el concepto del sistema de innovación, en el que el proceso de innovación se dan diferentes interacciones entre las etapas de invención y desarrollo del producto o servicio antes de que la innovación sea exitosamente incorporada y difundida en los mercados.

Las políticas de "tercera generación" están llamadas a atender los requerimientos que impone una economía basada en el conocimiento y de innovación generalizada. La política de innovación debe ser tratada como una política horizontal pero al mismo tiempo como una política específica, lo cual requiere de formas de análisis y acción que incorporen el tema deinnovación en distintas áreas de la política pública. 
8. Con profundas limitaciones, en la actualidad las PYME representan un estrato muy importante en la estructura productiva de la economía, ya que tienen una participación ampliamente mayoritria en los sectores productivos más importantes de la economía nacional, constituyéndose en algunas zonas del interior del país como la única forma de organización empresarial existente sobre la cual gira la actividad económica.

9. La economía peruana enfrenta severas restricciones, tanto en infraestructura como en la calidad del capital humano, lo cual conduce a plantear que para seguir creciendo necesita aumentar la inversión y mejorar la calidad de la educación.

10. El Perú cuenta con políticas favorables al sistema de innovación orientadas a la creación de organizaciones, pero que en términos de ejecución de funciones es completamente ineficaz.

11. Hay gran desconocimiento de la conducta innovadora de las empresas siendo necesario el levantamientos de datos sobre innovación, lo cual permitirá evaluar la operatividad de los instrumentos de política de innovación.

12. El sistema de innovación en Perú presenta muchas debilidades, destacándose la falta de una política integral de innovación, la inercia organizacional e institucional, con una limitada gestión del conocimiento.

13. Las empresas orientadas al mercado externo están empezando a incorporar a la innovación como una estrategia de negocios de mediano y largo plazo.

14. Hay clara falta de toma de conciencia de parte de las autoridades peruanas de la urgente necesidad de articulación de la política de competitividad con las políticas sectoriales, lo que conduce prioritariamente a las grandes reformas pendientes relacionadas con la reforma educativa, industrial y la del sistema judicial.

\section{LITERATURA CITADA}

Arbulú, J. (2006). "La PYME en el Perú”. En: PAD, revista de los egresados de ESAN. Diciembre del 2006. Lima.

Banco Central de Reserva del Perú (BCRP). (2010). Limitantes al crecimiento económico de largo plazo en el Perú. BCRP, Lima.
Banco Internacional de Reconstrucción y Fomento (BIRF) y Banco Mundial (BM). (2010). Doing Business 2011. Creando oportunidades para los emprendedores. Banco Mundial y la Corporación Financiera Internacional, Washington.

Cañizales, Andrés y Lugo, Jairo. (2007). "Telesur: estrategia geopolítica con fines integraciones". En: ConFines. №3/6. Agosto-diciembre 2007.

CEPAL, UNCTAD. (2012). Examen de las políticas de ciencia, tecnología e innovación del Perú. Ginebra, CEPAL, UNCTAD.

Contreras Álvarez, Gerardo. (2002). "La globalización económica desde los paradigmas del mercado y la solidaridad humana". En: InterSedes: Revista de las Sedes Regionales. (2002). Vol. III, Núm. 4, pp. 155-169. Universidad de Costa Rica, Costa Rica.

Díaz, J. y Kuramoto, J. (2010). Evaluación de políticas de apoyo a la innovación en el Perú. GRADE, Lima.

Espina Montero, A. (2004). "Sobre estabilidad de precios, deflación y trampas de liquidez en el G-3". En: Documentos de Trabajo. $\mathrm{N}^{\circ} 3$, 2004. Madrid, Real Instituto Elcano de Estudios Internacionales y Estratégicos. Fecha de consulta: 30/11/2012. Disponible en: $<$ http://www.realinstitutoelcano.org/documentos/84/84.pdf>.

Fernández Aceituno. (2009). Gestión en tiempos de crisis. Editorial Deusto S.A., Asturias, España. Fecha de consulta: 20/08/2012. Disponible en: < http:/www.casadellibro.com/ leer?li=1\&isbn $=9788423427819>$.

Gamboa, Franco y Alcocer, Pamela. (2012). "Brasil: entre el espejismo y el oasis”. En: Periódico Mensual Pukará. Año 6, N70. Qollasuyu, Bolivia. Fecha de consulta: 20/10/2012. Disponible en: <http://www.periodicopukara. com/archivos/pukara-70.pdf > .

Gandarilla Salgado, José Guadalupe. (2007). “iDe qué hablamos cuando hablamos de globalización? Una incursión metodológica desde América Latina”. En: Revista Ánfora. Año 14, $\mathrm{N}^{\circ} 22$, enero-junio 2007. Universidad Autónoma de Manizales, Colombia.

Global Entrepreneurship Monitor (GEM). (2009). Empreendedorismo no Brasil. Instituto Brasileiro da Cualidade e Productividade, Curitiba. 
Godet, Michael. (2007). Prospectiva estratégica: problemas y métodos. Cuadernos de LIPSOR $\mathrm{N}^{\circ} 20$. Prospektiker - Instituto Europeo de Prospectiva y Estrategia, Donostia.

Guidice B., Víctor. (2010). PYMEs e innovación en el Perú. UNMSM, Lima.

Huerta Moreno, Guadalupe. (2013). Financiarización, crisis e ingobernabilidad. UAMA, Colombia.

Krugman, P. (2009). El retorno de la economía de la depresión y la crisis actual. Huertas Industrias Gráficas SA., Madrid.

Krugman, P. (2012). Detengamos esta crisis ya. Editorial Paidós Mexicana, SA., México DF.

Martin Fragachán, Gustavo. (2011). "Cuando el presente nos alcance... Globalización: nuevas tecnologías, estrategia y comunicación política”. En: Orbis, revista científica de ciencias humanas. Septiembre-diciembre 2011.

Mayorga, D., y García, E. (2010). "La innovación empresarial en Perú". En: INCAE Business Review. Vol. 1, N¹0, enero-abril 2010.

Mercado Pacheco, Pedro. (2010). "Globalización y crisis económica cla resurrección del Estado?". En: IUS, revista del Instituto de Ciencias
Jurídicas de Puebla. №25. Fecha de consulta: 14/09/2012. Disponible en: <http://www.redalyc.org/articulo.oa?id=293222977016> .

Pascual, Rodrigo F. y Giotto, Luciana. (2010). "Reconceptualizando lo político: Estado, mercado mundial, globalización y neoliberalismo". En: Argumentos. $\mathrm{N}^{\circ} 23$. Septiembre-diciembre 2010. Fecha de consulta: 21/03/2013. Disponible en: <http://www.redalyc.org/articulo. oa id $=59518491006>$.

Stiglitz, Joseph E. (2012). El precio de la desigualdad. Santillana Edic. Generales, España.

Villarán, F. (2010). Las PYMEs en la estructura empresarial peruana. Servicios para el Desarrollo (SASE), Lima.

Villarán, F. (2012). “Articulación y asociatividad de las PYMEs peruanas". Ponencia presentada en el Encuentro de PYMEs de Iberoamérica y África del Norte (SEGIB). Octubre del 2012. Fecha de consulta: 30/11/2012. Disponible en: <http:// segib.org/actividades/files/2012/10/FernandoVillaran.pdf $>$.

Yory, Carlos Mario. (2012). “El grupo BRIC y el 'Efecto Roquefort': una aproximación a la noción de región-emergente en el contexto de la globalización”. En: GEOgraphia. Vol. 14, N⒉ 